\title{
Civilisations
}

Revue internationale d'anthropologie et de sciences

humaines

52-1 | 2004

Transmission des savoirs et interactions culturelles

\section{La néolithisation de la zone balkano-danubienne et l'occupation du territoire}

Janusz K. Kozłowski

\section{OpenEdition}

Journals

Édition électronique

URL : http://journals.openedition.org/civilisations/733

DOI : $10.4000 /$ civilisations.733

ISSN : 2032-0442

\section{Éditeur}

Institut de sociologie de l'Université Libre de Bruxelles

\section{Édition imprimée}

Date de publication : 1 mai 2004

Pagination : $9-24$

ISSN : 0009-8140

Référence électronique

Janusz K. Kozłowski, « La néolithisation de la zone balkano-danubienne et l'occupation du territoire », Civilisations [En ligne], 52-1 | 2004, mis en ligne le 27 mai 2009, consulté le 02 juin 2020. URL : http:// journals.openedition.org/civilisations/733 ; DOI : https://doi.org/10.4000/civilisations.733 


\title{
La néolithisation de la zone balkano-danubienne et l'occupation du territoire
}

\author{
JANUSZ K. KOZKOWSKI
}

\section{LES HABITATS MÉSOLITHIQUES À L'AUBE DE LA NÉOLITHISATION}

Le problème fondamental quant à la néolithisation de l'Europe est celui de l'interaction entre les populations mésolithiques locales et les populations exogènes qui ont apporté les éléments de l'économie de production et de nouveaux modes de vie comme par ex. : plantes cultivées et animaux domestiques.

Malgré les progrès récents des recherches sur le Mésolithique des Balkans et dans le bassin du moyen Danube, le nombre des sites de cette période n'a pas augmenté. Les découvertes de quelques sites mésolithiques dans la Grande Plaine hongroise, entre le Danube et la Tisza (Kértesz 1993, 1994), ont été considérées comme des arguments en faveur d'un peuplement mésolithique assez dense à l'aube de la néolithisation. Ce peuplement, d'après certains chercheurs (par ex. : Chapman 1994, Budia 1999), pouvait constituer un réseau d'habitats suffisamment dense pour assurer la diffusion des éléments de la nouvelle économie et de modes de vie, peut-être même sans immigration de nouveaux groupes humains originaires du sud-est. Cette constatation nous paraît néanmoins encore prématurée, aucun des sites trouvés entre le Danube et la Tisza n'est daté au Mésolithique récent. Il s'agit de sites qui représentent l'Epigravettien (peut-être avec certains éléments sauveterriens), datés du Préboréal et du Boréal, donc du Mésolithique ancien. Les datations pour les sites de Jaszbéreny (Kértesz et al.1994) qui sont plus récentes que $6800 \mathrm{BC}$ ont été obtenues à partir de carbonates, nettement épigéniques. Quelques trapèzes trouvés en surface de site de Jaszbéreny n'appartiennent pas non plus à ces industries mésolithiques, mais sont liés à la Céramique Linéaire orientale, dont les tessons sont présents également en surface. Notons aussi la présence à Jasztélék de fondations d'une tente légère, reposant sur des poteaux inclinés vers le centre (Fig. 1). Cette construction ressemble aux structures d'habitat circulaires connues du Mésolithique de la Grande Plaine de l'Europe du Nord, mais diffère considérablement des maisons quadrangulaires en poteaux et argile néolithiques des Balkans et du bassin danubien (Kértesz 1996).

Puisque dans les Balkans et dans le bassin du moyen Danube il n'existait pas de réseau d'habitats mésolithiques suffisamment dense et continu pour assurer la dif- 


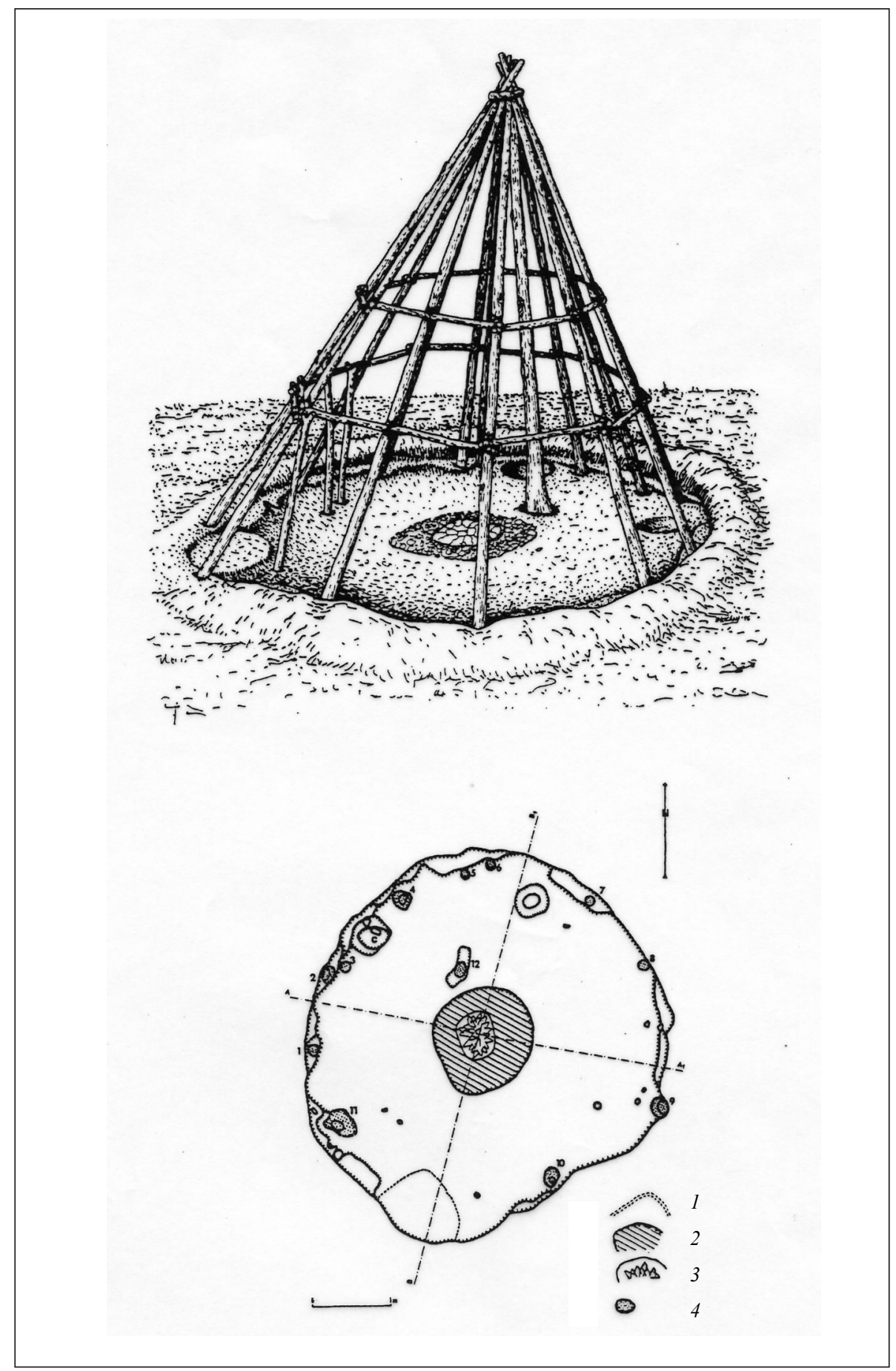

Fig. 1

Jasztélék (Hongrie). Reconstitution d'une hutte (d'après Kértesz 1996). 1.- limites de la structure, 2 et 3.-foyer, 4.- trous de poteau. 
fusion de la nouvelle économie, il est beaucoup plus probable que la néolithisation de cette zone résulte d'un apport migratoire extérieur. Notons que les habitats du Mésolithique récent forment seulement quelques concentrations isolées, par ex. dans le Peloponnèse, au Monténégro, dans la région de Portes de Fer et éventuellement dans le sud de la Transdanubie (Kozłowski, Kozłowski 1984) (Fig. 2).

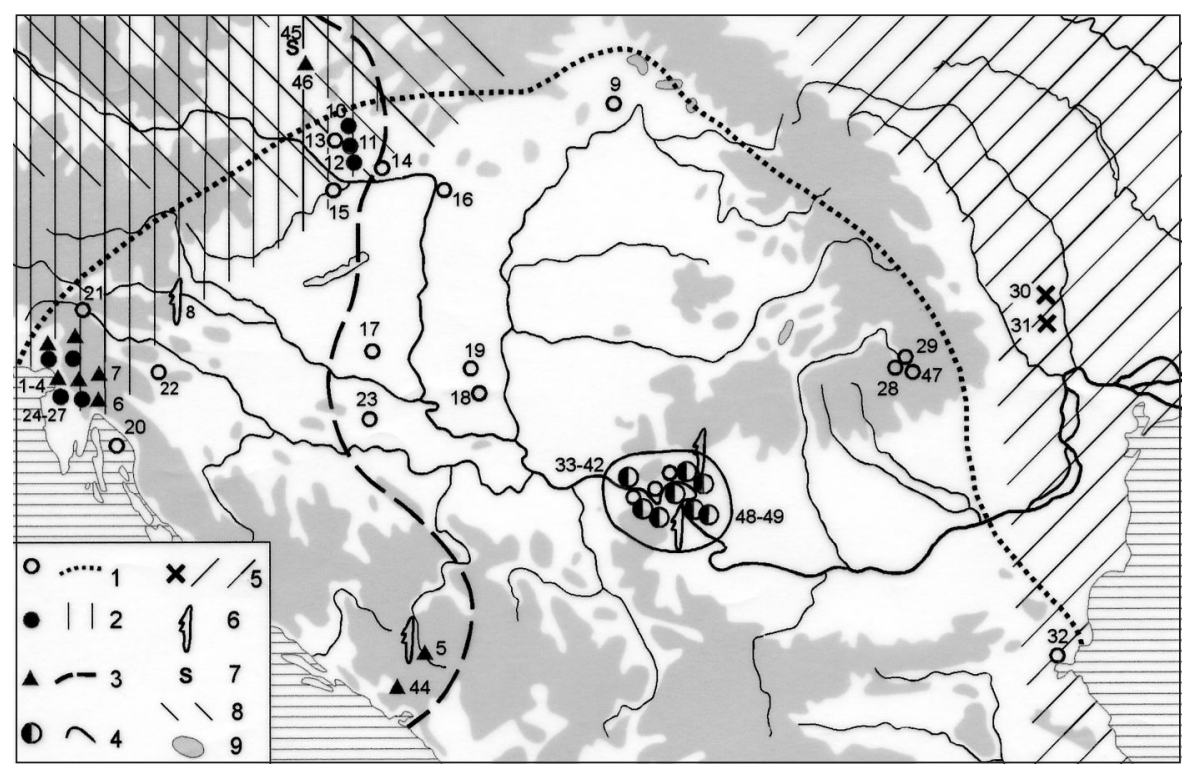

Fig. 2

Carte du Mésolithique du bassin danubien publiée par J. K. Kozłowski et S. K. Kozłowski en 1984. Entités taxonomiques : 1.-Epigravettien, 2.- Sauveterrien, 3.-Castelnovien, 4.- culture de Lepenski Vir, 5.- type Malusteni, 6.- sites avec harpons plats, 7.- type de Sakvice, 8.- Carpates.

Liste de sites : 1.- Grotta Azzurra, 2.- Grotta Benussi, 3.- Grotta della Tartaruga, 4.- Grotta Zingari, 5.-Odmut, 6.-Pod Crmukljom, 7.-Dedkov Trebez, 8.-Spehovka, 9.-Streda nad Bodrogom, 10.-Sered, 11.-Mostova, 12.-Tomasikovo, 13.-Dolna Streda, 14.-Hurbanovo, 15.-Gyor, 16.-Szodliget, 17.-Pecs, 18.- Bacska Polanka, 19.- Hajdukovo, 20.- Kopacina Pecina, 21.- Polijsiska Cerekev, 22.-Vindja, 23.-Rusevo, 28.- Gilma, 29.-Cremenea, 30.-Malusteni IV, 31.-Beresti, 32.-Dekilitaz, 33.-VeteraniTerasa, 34.- Icoana, 35.- Razvrata, 36.- Ostrovul Banului, 37.- Schela Cladovei, 38.- Padina, 39.-Lepenski Vir, 40.-Vlasac, 41.-Hajducka Vodenica, 42.-Alibeg, 44.- Crvena Stijena, 45.- Sakvice, 46.- Mikulcice, 47.- Costanda Laudauti, 48.- Ostrovul Corbului, 49.- Ostrovul Mare. (Etat de cette liste en 1982).

Le meilleur exemple est donné par le Mésolithique en Grèce. Les rares sites mésolithiques sont connus des grottes qui voisinent avec les plaines alluviales du littoral, surtout égéen. Le nombre restreint de ces sites contraste avec la présence de nombreux habitats du Néolithique ancien, surtout sur la Plaine de Thessalie aux alentours de Larissa (Van Andel, Runnels 1995). Les sites d'Argolide nous ont fourni les preuves des interactions entre les populations mésolithiques et néolithiques. Le premier exemple concerne la grotte de Franchthi où la phase finale du Mésolithique (phase X d'après C. Perlès, 1990), datée autour de 5900 BC, s'inscrit dans la continuité technologique des industries mésolithiques précédentes, mais, en même temps, a connu les premières plantes domestiquées (surtout les céréales, puisque les légumineuses ont 
été récoltées à l'état sauvage par les populations paléo-mésolithiques et que, d'après J. Hansen [1991] il ne s'agit pas d'une domestication locale de ces plantes) (Fig. 3).

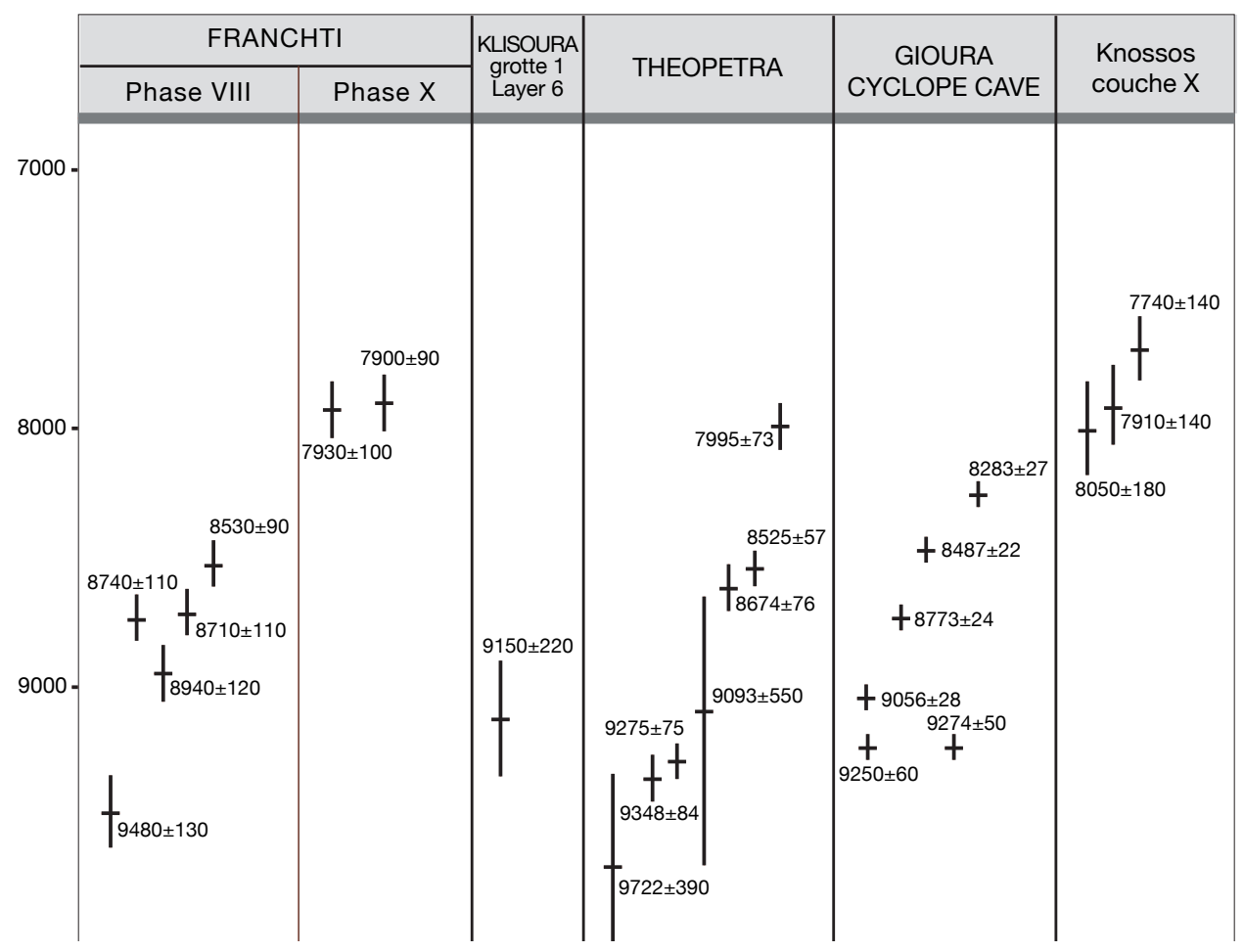

Fig. 3

Synchronisme entre les différents niveaux du Mésolithique en Grèce et ceux avec économie néolithique mais sans céramique

(Franchthi phase X, Knossos couche X).

Toutes les dates sont en B.P. non calibré.

Un vrai hiatus apparaît à Franchthi autour de 5800 ans $\mathrm{BC}$ avec la fondation d'une colonie néolithique en dehors de la grotte (dans le lieu dit « Paralia ») avec une céramique bien développée (Vitelli 1993) et avec des industries macrolaminaires en silex et en obsidienne importés. Ces industries macrolaminaires indiquent nettement la présence de nouvelles populations et n'ont rien de commun avec la tradition technologique du Mésolithique balkanique. D'ailleurs, ces industries sont apparues parfois sans céramique (ou plutôt avec une céramique assez rare), comme dans le cas des niveaux de base d'Otzaki Magula en Thessalie (Milojčić et al. 1962) et, pour cette raison, furent attribuées au Néolithique précéramique. Comme l'a bien remarqué C. Perlès (1987), les techniques macrolaminaires sont diagnostiques pour le Néolithique ancien avec céramique.

Un autre exemple de l'interaction entre le Mésolithique et le Néolithique est fourni par la séquence de la Grotte $n^{\circ} 1$ de Klisoura, en bordure de la Plaine d'Argolide. Dans cette grotte, au sommet de la séquence du Mésolithique (couche 3), dans le contexte des industries à éclats sur radiolarites et chailles locales, ont été trouvés quelques 
objets de caractère macrolaminaire (par ex. : un fragment de grande lame transformé en pièce esquillée, un grand éclat outrepassé détaché d'un nucléus à lames à un plan de frappe) en silex «blond » importé probablement des Balkans du Nord (Koumouzelis et al. 1996). Il s'agit probablement des objets acquis par les groupes mésolithiques auprès des habitants de colonies du Néolithique ancien de la Plaine d'Argolide. En effet, les niveaux les plus anciens du Néolithique de Lerna et de Dendra ont fourni des industries macrolaminaires avec nombreux objets en silex «blond » et en obsidienne (Kozłowski et al. 1996).

En Thessalie, le contraste entre les nombreux sites du Néolithique ancien - généralement les niveaux de base de tells - et avec un seul site du Mésolithique dans la grotte Theopetra est encore plus net (Kyparissi-Apostolika 1999). Malheuresement le contenu des couches mésolithiques de cette grotte est difficile à identifier, à cause de perturbations des sédiments. Les couches qui ont fourni les datations contemporaines $\mathrm{du}$ Mésolithique contenaient des industries macrolaminaires proches de celles du Néolithique de la Plaine de Larissa, associées à des outils sur éclat peu diagnostiques, parfois mélangés, ainsi qu'à des outils épigravettiens semblables à ceux des niveaux sous-jacents (Adam 1999).

Contrairement à l'opinion de M. Budia (1999), j'hésiterai à voir dans la séquence de la grotte de Theopetra une transformation locale du Mésolithique au Néolithique, en association avec une céramique très primitive.

\section{LA QUESTION DU MÉSOLITHIQUE/NÉOLITHIQUE DE LA RÉGION DE PORTES DE FER}

Le problème de transition Mésolithique/Néolithique dans la région de Portes de Fer constitue un autre sujet de controverses. Malgré une rupture nette dans les séquences stratigraphiques de Lepenski Vir et de Padina entre les niveaux mésolithiques et néolithiques du point de vue de l'économie (la chasse, la pêche et seulement le chien domestique dans les niveaux mésolithiques, en contraste avec les animaux domestiques [chèvre/mouton, bœuf et suidés] et les céréales cultivées dans les niveaux néolithiques), certains chercheurs continuent à voir dans le cas de Lepenski Vir une continuité entre le Mésolithique et le Néolithique dans la région de Portes de Fer. Pourtant, ce hiatus entre le Mésolithique et le Néolithique est bien marqué dans la technologie : le Néolithique ancien avec céramique de Starčevo commence avec l'introduction de la technique macrolaminaire et la dominance du silex mésozoïque de la Plate-forme Pré-Balkanique ; ces deux phénomènes, nous les observons aussi bien à Lepenski Vir (couche III) qu'à Padina (unité B) (Kozłowski, Kozłowski 1984 ; Radovanović, 1998). Le seul élément de continuité entre le Mésolithique et le Néolithique relève de l'architecture en pierre : les maisons trapézoidales et certains foyers structurés, sont connu également dans les niveaux néolithiques à Padina et à Lepenski Vir (Radovanović, 1998, Garašanin, Radovanović 2001). J'hésiterais néanmoins à attribuer toutes les constructions trapézoïdales de Lepenski Vir au Néolithique, comme l'avait suggéré récement D. Borić (1999). Dans le cas de Lepenski Vir et de Padina, nous aurions donc une continuité architecturale : les populations néolithiques ont repris aux derniers pêcheurs-chasseurs mésolithiques les maisons à soubassements de pierre (différentes des maisons en torchis et clayonnage bien connues dans la culture 
de Starčevo), un signe particulier de la sédentarisation des groupes mésolithiques. Par contre l'art mésolithique de Lepenski Vir, avec certains motifs liés à l'eau et aux poissons (Radovanović 1997) - par ex. les motifs de lignes ondulées et les têtes anthropomorphes à bouche de poisson - n'a pas été assimilé par les groupes néolithiques. Notons que nous avons quelques preuves qu'avant l'apparition des groupes Starčevo dans la zone de Portes de Fer, il y avait des contacts entre les populations mésolithiques et celles de Starčevo, en Serbie orientale et en Olténie, donc dans les régions qui entourent la zone de Portes de Fer. Ces contacts sont attestés, par exemple, par les importations d'objets en silex de la Plate-forme Pré-Balkanique, dès la phase Lepenski Vir I. La contemporaneité des deux populations est confirmée par les datations C-14 pour la période entre 5300 et $5700 \mathrm{BC}$ (Fig. 4). Les fragments de céramique dans les niveaux Lepenski Vir Ib et II, s'ils ne sont pas intrusifs, pourront éventuellement confirmer aussi les contacts entre les groupes mésolithiques et les populations de la culture de Starčevo ( $c f$. Borić 1999).

L'exemple des sites de la région de Portes de Fer montre donc une acculturation des populations mésolithiques due aux contacts de plus en plus intenses avec les agriculteurs indigènes de la culture de Starčevo.

Nous avons montré ici les différentes modalités des interactions entre les populations exogènes néolithiques et les groupes locaux mésolithiques. Compte tenu du fait qu'aussi bien dans les Balkans que dans le bassin du moyen Danube les populations mésolithiques ont été peu nombreuses, distribuées seulement dans quelques microrégions limitées (comme par ex. l'Argolide, la vallée du Drin au Monténégro, la région de Portes de Fer) et assez diversifiées du point de vue culturel (Epigravettien dans l'Est de Balkans et dans le Bassin du moyen Danube, Castelnovien à l'Ouest de Balkans et industries particulières sur le littoral de la Mer Noire et dans le bassin du Danube inférieur). Le rôle joué par ces populations dans le processus de néolithisation était plutôt secondaire. Par contre, les groupes exogènes du Néolithique ancien balkanique, beaucoup plus nombreux et très homogènes du point de vue culturel, ont joué un rôle décisif dans la néolithisation de la zone balkano-danubienne.

\section{LE NÉOLITHIQUE ANCIEN BALKANIQUE - CULTURES AVEC CÉRAMIQUE PEINTE}

Le complexe à Céramique Peinte du Néolithique ancien balkanique (surtout dans la phase ancienne à céramique peinte en blanc) est très homogène sur un vaste territoire des Balkans de l'Est, de l'Argolide au Danube, et même plus au Nord, jusqu'en Olténie et en Transylvanie (Fig. 5). Cette entité est caractérisée par une économie basée sur la culture des céréales et légumineuses, l'élevage du mouton et de la chèvre associé à celui des suidés et des bovidés (bien que localement, par ex. en Voïvodine, le rôle de la chasse et de la pêche puisse être plus important). La technologie lithique est également très homogène (macrolaminaire, avec des outils à retouches marginales) et basée sur des matières premières lithiques provenant surtout de la Plate-forme Pré-Balkanique (Gatsov 1993). Les concrétions de silex de cette zone ont été décortiquées dans des ateliers (encore peu connus) et les nucléus ont été exploités au fur et à mesure des besoins pour en tirer des lames. Par conséquent, nous n'avons presque pas de produits de débitage dans les sites : nous trouvons uniquement des lames ou des 


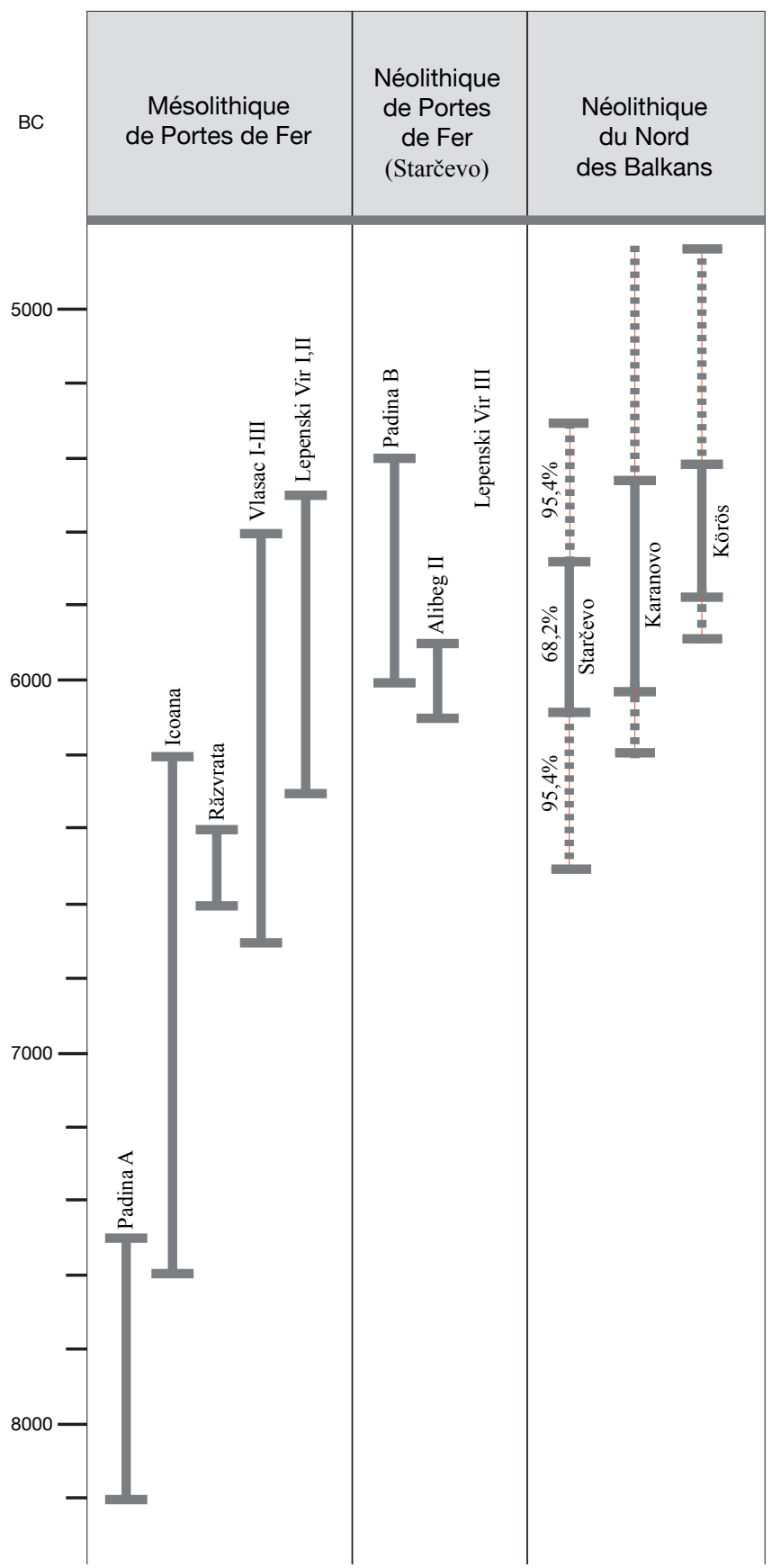

Fig. 4

Datations par C-14 des séquences mésolithiques et néolithiques de la région de Portes de Fer et des cultures néolithiques des Balkans du Nord

(moyennes des dates B.P. non calibré). 
outils très usés, ainsi que de rares nucléus très réduits. Les produits macrolaminaires en « silex balkanique » (et peut-être aussi en obsidienne, au début d'origine égéenne, plus tard carpatique) ont pu avoir une signification non seulement fonctionnelle, mais peut-être aussi sociale ou symbolique. Cette fonction pourrait être confirmée par les dépôts des lames réalisées dans ce silex, trouvés dans des vases ou des boîtes en argile cuite (par ex. à Nea Nikomedeia ou à Lepenski Vir III).

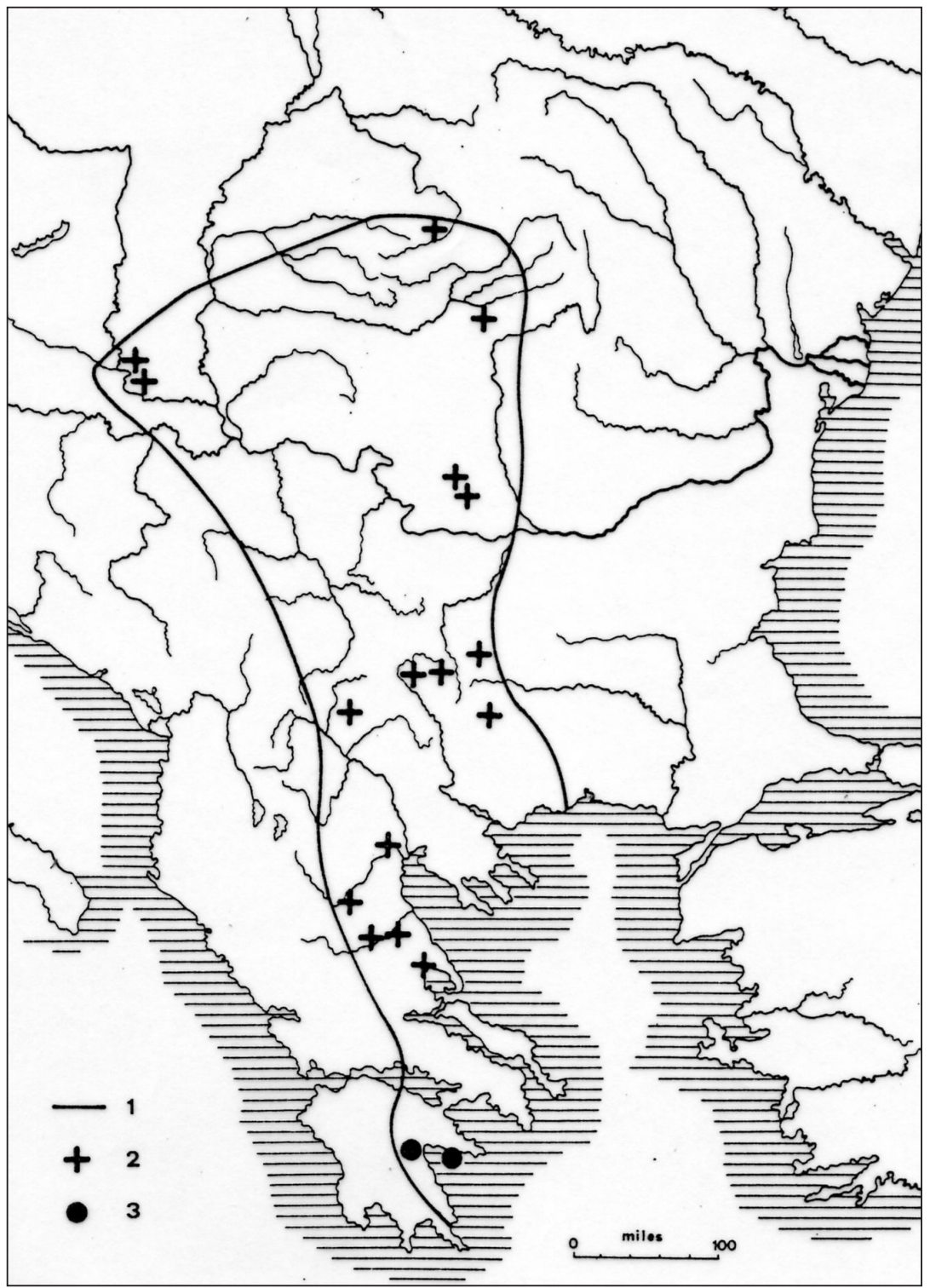

Fig. 5

Extension maximale du premier horizon du Néolithique ancien balkanique avec la céramique peinte en blanc: 1.- extension maximale, 2.- sites à céramique peinte en blanc (Protosesklo, Protostarcevo ou Gura Bacului), 3.- sites avec la céramique du type rainbow. 
La céramique, dont les motifs sont d'abord peints en blanc sur le fond rouge ou foncé, devient plus tard polychrome dans les différents complexes du Néolithique ancien, comme à Karanovo, Chavdar, Starčevo, Körös, etc. Elle est accompagnée d'une poterie grossière - dont le pourcentage varie d'un site à l'autre - et aussi d'une céramique monochrome à surfaces rouges ou brunâtres. Cette dernière était à l'origine de l'hypothèse de l'existence d'une phase à céramique uniquement monochrome, précédant la céramique peinte en blanc. En fait, cette hypothèse n'est basée que sur les deux sites bulgares de Kraïnitsi et de Koprivets (Stefanova 1996) ; il pourrait s'agir dans ce cas de différences fonctionnelles qui influençaient le répertoire céramique ou de différences régionales.

En Grèce, l'architecture de ces plus anciens complexes balkaniques est assez différenciée dès le début : nous observons la présence de structures rectangulaires avec fondations en pierre (par ex. à Lerna), mais aussi de nombreuses maisons en torchis et clayonnage, renforcées parfois de quelques poteaux, répandues surtout dans le nord des Balkans et dans le bassin danubien (Achilleon, Elechnitsa, Slatina, Karanovo).

Cette tradition du Néolithique ancien balkanique pénètre profondément dans le bassin de la Tisza, pour atteindre les piémonts des Carpates en Ukraine Transcarpatique. C'est dans le bassin de la Tisza que nous observons la transformation du complexe à Céramique Peinte balkanique en nouvelle entité - culture de la Céramique Linéaire orientale (Kalicz, Makkay 1977). En Hongrie, cette transformation est marquée par le groupe de Szatmar qui donne son origine à la Céramique Linéaire orientale (Kalicz 1983). Les découvertes récentes de sites tels que Slavkovce, dans la Plaine de la Slovaquie orientale, montrent que l'aire de la formation de cette nouvelle entité était beaucoup plus large et dépassait le nord-est de la Hongrie vers les Carpates (Kozłowski éd. 1997). Bien que la Céramique Linéaire présente de nouvelles techniques de décoration et de nouveaux motifs, l'économie, l'architecture et l'implantation des sites sont hérités du Néolithique ancien balkanique (Fig. 6). Ainsi, par exemple, les maisons rectangulaires aux parois en torchis et clayonnage dominent, bien qu'elles soient plus fréquemment armées de poteaux. Leurs dimensions dans le groupe de Szatmar sont de 7-9 x 12 m (par ex. à Mezoköved), de 5 x 9 m dans le linéaire classique (par ex. à Krasznokvajda) ou de 4 x 6,3 m, dans le cas de maisons bien conservées après la destruction, à Šarišske Michalany (Šiška 1998).

La population qui a produit la Céramique Linéaire orientale s'était mieux adaptée aux sources locales de matières premières, en premier lieu à l'obsidienne carpatique de Plateau de Zemplen-Tokai et aux limnoquartzites locaux. Les dernières traces de silex balkanique, nous les observons dans le site de Méhtelek qui se place à la limite de la culture de Körös et du groupe de Szatmar (Starnini 1994). Plus tard, avec l'importance croissante de l'obsidienne et des limnoquartzites, les chaînes opératoires complètes apparaissent dans des sites de différentes phases du Linéaire oriental (Kozłowski 1998).

Malgré ces adaptations aux conditions locales, les populations du linéaire oriental n'ont jamais pu franchir l'arc des Carpates, bien qu'elles aient pénétré profondément les vallées de rivières telles que la Topla, l'Ondava et le cours supérieur de la Tisza. Cette implantation du Linéaire oriental dans des environnements aussi différents que la Plaine de Slovaquie orientale et le piémont des Carpates a contribué à la création de nombreux groupes régionaux à l'intérieur de la culture à Céramique Linéaire orientale (Šiška 1989). C'est probablement une forte tradition du Néolithique ancien 
balkanique qui a limité les possibilités adaptatives des groupes du Linéaire oriental ; ces derniers n'ont jamais pu atteindre une extension aussi grande que les groupes du Linéaire occidental (Rubané).

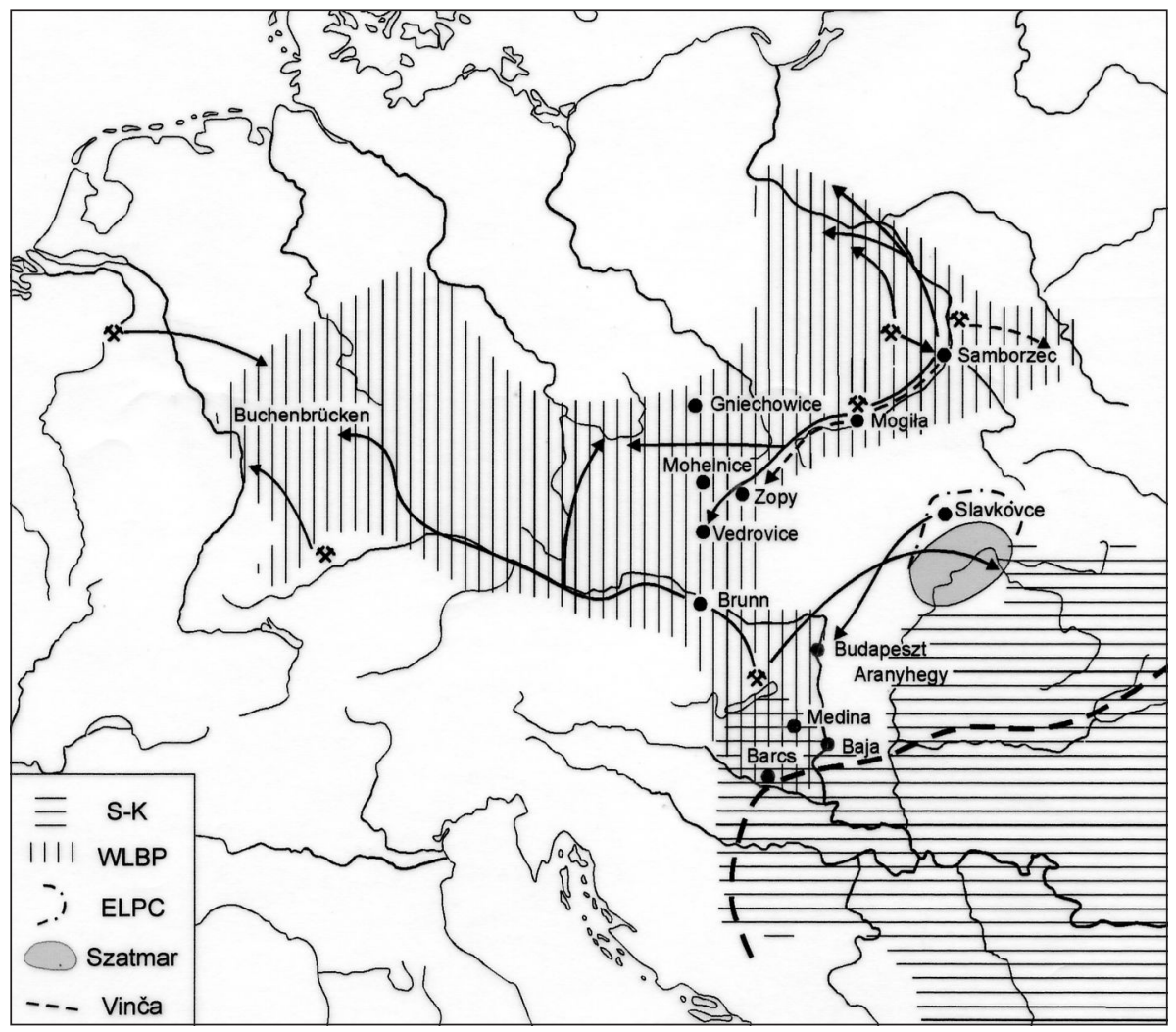

Fig. 6

Carte de l'Europe centrale pendant la diffusion des complexes linéaires oriental et occidental (Rubané). $S$-K-Starčevo-Körös phase finale, WLBP-Rubané, phase ancienne, ELPC-complexe linéaire oriental-phase ancienne.

Bien que le Linéaire oriental ait dépassé la zone néolithisée par les cultures à Céramique Peinte (surtout en Slovaquie orientale), il n'existe aucune preuve de contacts entre les groupes linéaires et le mésolithique local. Les trapèzes qui apparaissent dans le linéaire oriental sont sans doute hérités de la tradition du Néolithique ancien balkanique (Starčevo-Körös).

\section{LE LINÉAIRE OCCIDENTAL ET L'EXTENSION DE LA NÉOLITHISATION SUR LES PLATEAUX DE L'EUROPE CENTRALE}

En même temps que l'apparition de la Céramique Linéaire orientale, nous observons en Transdanubie et en Basse Autriche, l'émergence du complexe Linéaire occidental (Rubané). Dans ce cas, le hiatus entre le Néolithique ancien balkanique 
et le Rubané est beaucoup plus marqué, aussi bien dans la céramique (absence de la céramique peinte et apparition de différentes formes de vases, ainsi que de différentes techniques de préparation de pâtes) que dans les autres domaines technologiques et économiques. Ce hiatus est marqué en particulier dans les industries lithiques (technologies à lames et à éclats, différentes des technologies macrolaminaires de la culture de Starčevo, utilisation des matières premières locales, surtout les radiolarites de la région de lac Balaton, dominance des outils à retouches transversales - grattoirs et troncatures - ainsi que des perçoirs qui remplacent les lames retouchées de Starčevo, etc.) et dans l'architecture (apparition des maisons longues à rangées de poteaux). Notons aussi que la plus ancienne Céramique Linéaire occidentale ne couvre qu'une petite région de la frange septentrionale de la culture de Starčevo, notamment la zone située au sud du lac Balaton. La plus grande partie du territoire du Rubané le plus ancien (Transdanubie au nord de lac Balaton, Basse Autriche, Moravie, Slovaquie du Sud-Ouest) est un territoire néolithisé pour la première fois par les groupes du Linéaire occidental. Si la partie méridionale de ce territoire était, comme nous l'avons déjà souligné, faiblement peuplée au Mésolithique récent, au contraire, les territoires de Moravie, de Slovaquie occidentale et de Bohème (ainsi que les régions situées plus au nord, telles que l'Allemagne et la Pologne, où nous connaissons également des sites du Rubané le plus ancien), ont connu les différentes entités du Mésolithique récent (par ex. Beuronien, Epigravettien, Post-Maglemosien, Janislawicien, etc.). L'homogénéité de la phase ancienne du Rubané (dans toutes les domaines de l'économie, comme ceux de la culture matérielle et symbolique) s'oppose donc à l'hétérogénéité du substrat mésolithique dans la partie nord de l'Europe Centrale. Des différences régionales importantes n'apparaissent que dans la phase rente du Rubané, principalement avec l'émergence de groupe de Želiezovce.

Ces constatations s'opposent aussi bien à l'hypothèse selon laquelle le complexe Linéaire occidental représenterait une acculturation des groupes mésolithiques locaux, dont le réseau aurait facilité la diffusion de la nouvelle économie et de la céramique, qu'à l'idée que le Rubané ne représenterait qu'un prolongement de la tradition du Néolithique ancien balkanique, modifiée par les conditions écologiques du bassin danubien (pour la discussion voir Gronnenborn 1999 et Tillmann 1993).

Les seuls liens de la céramique rubanée avec celle de Starčevo sont certains types de céramique grossière à décors imprimés on plastiques ; par contre la phase ancienne du Rubané montre plusieurs éléments inconnus du Starčevo : formes de vases carenées, profilation parfois accentuée, décors cannelés. Ces éléments sont bien connus dans le complexe de Vinča qui apparaît dans le nord des Balkans autour de 5600 BC, donc dans la même période que le Rubané le plus ancien (dont les datations varient entre 5600 et $5000 \mathrm{BC}$ ). Les changements technologiques et stylistiques dans les industries lithiques du Rubané ancien par rapport au Néolithique ancien balkanique trouvent également des analogies dans les industries lithiques du complexe de Vinča. Dans l'architecture, nous observons également des analogies entre les maisons à poteaux du Rubané le plus ancien et celles de Vinča, surtout, dans les plans allongés et les parois extérieures en forme de palissades continues (bien connues des plus anciens sites du Rubané par exemple à Brunn en Basse Autriche - Lenneis 1997).

Les recherches récentes dans la zone de la Mer Marmara et la Thrace orientale montrent, que le complexe de Vinča n'est pas une simple conséquence de l'évolution du Néolithique ancien balkanique, comme le suggèrent actuellement plusieurs 
chercheurs, mais que les influences anatoliennes ont joué un rôle important dans sa genèse.

Ce point de vue, qui a déjà été exprimé au début des recherches sur le complexe de Vinča, pourrait être justifié par les éléments suivants :

a. les racines de la technologie céramique de Vinča apparaissent en Anatolie, surtout dans le nord-ouest de cette zone, bien avant le début du complexe de Vinča dans les Balkans du Nord. M. Özdögan et N. Baseglen (1999) parle d'une « common developping zone » entre le Nord-Ouest de l'Anatolie et le Nord-Est des Balkans. Cette technologie céramique présente des analogies avec les complexes énéolithiques de l'Anatolie centrale et orientale.

b. les changements importants dans la technologie lithique ont eu lieu entre le Néolithique ancien balkanique et le complexe de Vinča. Il s'agit de l'abandon de la technologie macrolaminaire effectuée en plusieurs épisodes dans les endroits différents au profit de technologies de production de lames plus petites et d'éclats comme effet de chaînes opératoires complètes, effectuées sur place dans les sites d'habitat. Les outils à retouches latérales ont été remplacés par ceux à retouches transversales (surtout les grattoirs, troncatures et perçoirs). Les changements technologiques, nous les observons dans la région de la Mer Marmara bien avant la formation du complexe de Vinča, par ex. dans la phase classique de la culture de Fikirtepe (Özdögan 1997, Gatsov 2000) et dans la séquence du site Hoca Cesme (sur le littoral de la Thrace turque), vers 6000-5900 BC. Cette nouvelle technologie est accompagnée dans cette zone par de la céramique foncée à profil plus accentué, évoquant la céramique de Vinča.

c. les sources de l'architecture de la culture de Vinča avec certaines maison à poteaux, peuvent se trouver également dans la région de la Mer de Marmara. Par exemple, le site d'Ilipinar, déjà dans sa phase $\mathrm{X}$ (vers $6000 \mathrm{BC}$ ), a fourni de petites maisons rectangulaires avec un rang intérieur de poteaux ; au contraire, dans les phases VII-VIII (5800 BC), apparaissent les grandes maisons rectangulaires avec des parois en forme de palissade. Ce n'est que dans la phase V que nous observons le retour de l'architecture en briques crues (5400 BC) (Rodenberg 1995).

Si la genèse du complexe culturel de Vinča est effectivement le résultat d'une nouvelle vague de population d'origine anatolienne cette fois, par la région de la Mer Marmara (en contraste avec la première vague diffusée par voie maritime transégéenne), nous pouvons supposer que cette vague a joué également un rôle dans l'origine du complexe Linéaire occidental.

Ce processus de genèse du Linéaire occidental pourrait expliquer les différences fondamentales qui opposent la diffusion de ce complexe à celle du Linéaire oriental. Le Linéaire oriental était une simple prolongation du Néolithique ancien balkanique, dont les possibilités adaptatives ont été limitées. Par contre, dans la formation du Linéaire occidental, un apport démographique nouveau et des possibilités adaptatives accrues grâce aux nouvelles technologies ont permis de conquérir les plateaux loessiques de l'Europe moyenne et même de pénétrer certaines régions de la Grande Plaine (Fig. 7).

Il est intéressant de ce point de vue, d'observer que les groupes orientaux du Rubané, après avoir suivi les vallées du Dniester, du Prut et du Seret à l'est de Carpates, sont apparus non seulement dans le bassin du Danube inférieur (Valachie), 


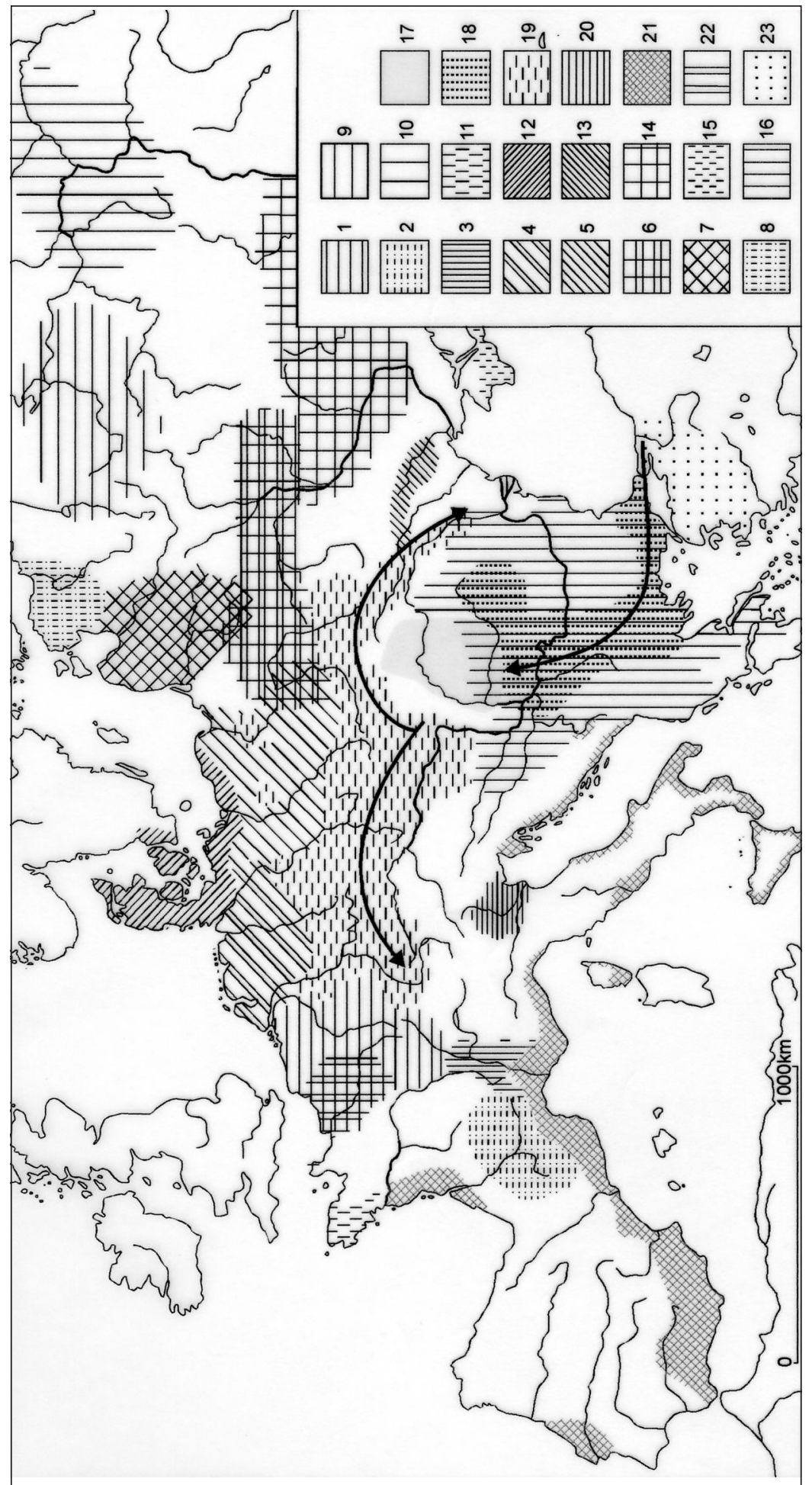

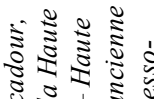

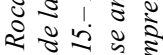
i. 虫 दิ

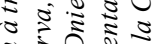
₹

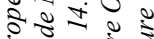

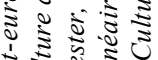
2 $\frac{2}{3} \cdot \frac{\pi}{2} \cdot$

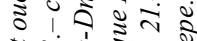
ॠ $\infty$ o.

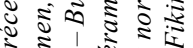

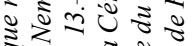
:

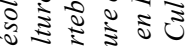
1. 1.200 $\because 2 \approx 100$ ป.

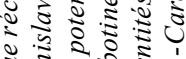

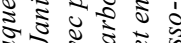

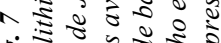

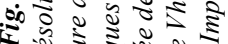

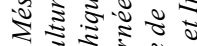
ร 3 ᄀ. 营

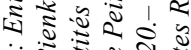

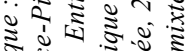

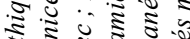

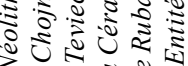

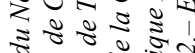

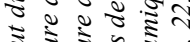

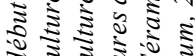

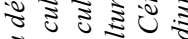
ส 1.1. ₹ 0.01. ป ฐ ฐ

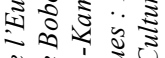
$\approx \approx$ 它

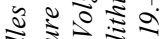

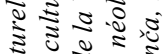

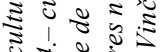

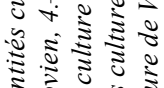
ปे ¿ 0.00 ن 
mais aussi dans la zone de la Mer Marmara. Cette présence est confirmée par la céramique rubanée qui apparaît dans la couche 3 de la grotte de Yarimburgaz, près d'Istanbul (Özdögan 1998). Cette apparition achève le mouvement du Rubané autour des Carpates pour arriver au point du départ de l'impact qui a provoqué l'origine du complexe de Vinča et qui a probablement contribué à l'origine du Linéaire occidental.

La direction occidentale de la diffusion du Rubané à travers le bassin danubien jusqu'à la Rhénanie, la Belgique et le Bassin Parisien a eu lieu dans les régions dotées d'un habitat mésolithique plus dense et, à la limite occidentale de son extension, a rencontré les premiers groupes du Néolithique d'origine méditerranéenne. C'est à cause de cela que le Rubané occidental est moins homogène que le Rubané oriental et présente à la frange occidentale des éléments aussi bien mésolithique que dérivant des entités du Néolithique ancien méditerranéen et/ou atlantique.

Janusz K. Kozłowski, Professeur à l'Université Jagellone de Cracovie.

\section{RÉFÉRENCE BIBLIOGRAPHIQUES}

ADAM E.

1999 Preliminary Presentation of the Upper Palaeolithic and Mesolithic Stone Industries from Theopetra Cave, Western Thessaly, in ADAM E., BAILEY G., PANAGOPOULOU E., PERLÈS C., ZACHOS K. (éds), The Palaeolithic Archaeology of Greece and Adjacent Areas, British School at Athens Studies, vol. $3: 266-270$.

BORIĆ D.

1999 Places that created time in the Danube gorges and beyond, c. 9000-5500 BC., Documenta Praehistorica $26: 41-70$.

BUDIA M.

1999 The transition to farming in Mediterranean Europe. An indigenous response, Documenta Praehistorica 26 : 119-141.

CHAPMAN J.

1994 The origins of farming in South-Eastern Europe, Préhistoire Européenne 6 : 133-156.

GARAŠANIN M., RADOVANOVIĆ I.

2001 A pot in house 54 at Lepenski Vir I, Antiquity 75 : 118-125. 


\section{GATSOV I.,}

1993 Neolithic chipped stone industries in Western Bulgaria, Krakow : Jagiellonian University, Institute of Archaeology : 54 p., 16 pl.

2000 Chipped stone assemblages from South Bulgaria and Nord-West Turkey (Epipalaeolithic/Mesolithic and Neolithic), in NIKOLOVA L. (éd.), Technology, Style and Society. Contribution to the innovation between the Alps and the Black Sea, Oxford: BAR International Series 858 : 1-12.

\section{GRONENBORN D.}

1999 A variation on a basic theme : the transition to farming in Southern Central Europe, Journal of World Prehistory 13/2: 123-210.

HANSEN J. M.

1991 The Palaeoethnobotany of Franchthi Cave. (Excavation at Franchthi Cave, Greece, fasc. 7), Bloomington-Indianapolis : Indiana University Press : 280 p., 23 pl.

\section{KALICZ N.}

1983 Die Körös-Starčevo-Kulturen und ihre Beziehungen zur Linien-bandkeramik, Nachrichten aus Niedersachsens Urgeschichte 52 : 91-130.

\section{KALICZ N., MAKKAY J.}

1977 Die Linienbandkeramik in der Grossen Ungarischen Tiefbene, Budapest : Akadémiai Kiadó : 385 p.

\section{KÉRTESZ R.}

1993 Data to the Mesolithic of the Great Hungarian Plain, Tisicum $8:$ 81-104.

1994 Late Mesolithic chipped stone industry from the site Jásztelek I (Hungary), in A körkortól a középkoring, Szeged : Szegedi Tudomanyegyetem : 23-39.

1996 At the fringes of three worlds. Hunter-gatherers and farmers in the middle Tisza Valley, Szolnok: Damjanich Muzeum : 75 p.

KÉRTESZ R., SÜMEGI P., KOZÁK M., BRAUN M., FÉLEGYHÁZI E., HERTELENDI E.

1994 Archaeological and Palaeoecological Study of an Early Holocene Area (Jaszbereny I), Acta Geographica Debrecina 32 : 5-49.

KOUMOUZELIS M., KOZŁOWSKI J.K., NOWAK M., SOBCZYK K., KACZANOWSKA M., PAWLIKOWSKI M., PAZDUR M.

1996 Prehistoric settlement in the Klisoura Gorge, Argolid, Greece, Préhistoire Européenne 8 : 143-173.

KOZŁOWSKI J.K.

1998 The lithic industry of the Eastern Linear Pottery Culture in Slovakia, Slovenska Archeologia 37-2 : $377-410$.

KOZŁOWSKI J.K. (éd.)

1997 The Early Linear Pottery Culture in Eastern Slovakia, Kraków : Polska Akademia Umięjetności : $282 \mathrm{p}$.

KOZŁOWSKI J.K., KACZANOWSKA M. PAWLIKOWSKI M.

1996 Chipped stone industries from Neolithic levels at Lerna, Hesperia 65/3 : 295-372.

KOZŁOWSKI J.K., KOZŁOWSKI S.K.,

1984 Chipped stone industries from Lepenski Vir, Preistoria Alpina 19 : 259-294.

KYPARISSI-APOSTOLIKA N.

1999 The Palaeolithic Deposits of Theopetra Cave in Thessaly (Greece), in ADAM E., BAILEY G., PANAGOPOULOU E., PERLÈS C., ZACHOS K. (éds), The Palaeolithic Archaeology of Greece and Adjacent Areas, British School at Athens Studies, vol. 3 : 232-239.

LENNEIS E.

1997 House forms of the Central European Linearpottery Culture and of the Balkan Early Neolithic - a comparison, Porčilo o raziskovaniu paleolitika, neolitika in eneolithica $v$ Sloveniji 24 : 143-150. 
MILOJČIĆ V., BOESSNECK J., HOPF M. (éds),

1962 Die deutschen Ausgrabungen auf der Argissa-Magula in Thessalien, I. Das präkeramische Neolithikum sowie die Tier- and Pflanzenreste, Beiträge zur ur- und frühgeschichtlichen Archäologie des Mittelmeer-Kulturraumes, 2. Bonn : Rudolf Habelt Verlag : 119 p., 29 pl.

\section{ÖZDÖGAN M.}

1997 The beginning of Neolithic economies in Southern Europe : an Anatolian perspective, Journal of European Archaeology 5/2 : 1-33.

1998 Anatolia from the Last Glacial Maximum to the Holocene Climatic Optimum : cultural transformations and impact of the environmental setting, Paléorient 23/2 :25-38.

ÖZDÖGAN M., BASGELEN N. (éds)

1999 Neolithic in Turkey. The Cradle of civilization. New Discoveries, Istanbul : Arkeoloji ve Sanat Yayinlari : $236 \mathrm{p}$.

PERLÈS C.

1987 Les industries du Néolithique « précéramique » Grèce : nouvelles études, nouvelles interprétations, Archaeologia Interregionalis : 19-39.

1990 Les industries lithiques taillées de Franchthi (Argolide, Grèce). T. II : Les industries du Mésolithique et Néolithique initial (Excavations at Franchthi Cave, fasc. 5), Bloominton-Indianapolis : Indiana University Press : $285 \mathrm{p}$.

\section{RADOVANOVIĆ I.}

1997 The Lepensli Vir Culture : a contribution to interpretation of its ideological aspects, in Antidoron Dragoslavo Srejovć completis LXV annis ab amicis, collegis, discipulis oblatum, Beograd : Centar za arheološka istraživanja Filozofskog fakulteta u Beogradu : 85-94.

1998 The Iron Gate Mesolithic, Ann Arbor, Michigan : International Monographs in Prehistory, Archaeological Series, vol. $11: 435 \mathrm{p}$.

ROODENBERG J. (éd.)

1995 The Ilpinar excavations I. Five Seasons of Fieldwork in NW Anatolia, Istanbul : Nederlands Historisch-Archaeologisch Instituut : vi $+180 \mathrm{p}$.

\section{STARNINI E.}

1994 Typological and technological analysis of the Körös Culture chipped, polished and ground stone assemblages of Méhtelek Nádas, Atti dela Società per la Preistoria e Protostoria della Regione FriuliVenezia Giulia 8 (1993) : 29-96.

\section{STEFANOVA T.}

1996 A Complex Analysis of Pottery from the Monochrome Early Neolithic Horizon and the Problem of Neolithization of Bulgaria, Poročilo 23 : 15-38.

ŠIŠKA S.

1989 Kultura s vychodnou linearnou keramikou na Slovensku, Bratislava : Veda Vydavatel'stvo Slovenskej Akadémie Vied : 208 p., 47 pl.

1998 Architektura neolitickej osady v šarišskich Michalanoch, Slovenska Archeologia 46/2 : 187-204.

\section{TILLMANN A.}

1993 Kontinuität oder Diskontinuität? Zur Frage einer bandkeramischen Landnahme im Südlichen Mitteleuropa, Archäologische Informationen 16/2 : 157-187.

VAN ANDEL T.H., RUNNELS C.N.

1995 The earliest farmers in Europe, Antiquity 69 : 481-500.

\section{VITELLI K.D.}

1993 Franchthi Neolithic pottery. Vol. I : Classification and Ceramic Phases 1 and 2 (Excavations at Franchthi Cave, Greece, fasc. 8), Bloomington-Indianapolis : Indiana University Press : 115 p. 